\title{
Real-World Electron Detector Performance in Scanning Electron Microscopes
}

\author{
Owen E. Healy and Richard B. Mott ${ }^{1}$ \\ 1. PulseTor LLC, 1580 Reed Road, Suite C2, Pennington NJ
}

We test the application of a novel figure of merit for electron detectors which is representative of their overall suitability for real-world imaging applications. Traditional performance numbers such as detective quantum efficiency (DQE) [1] and bandwidth each address single aspects of detector performance whereas efficiency, speed, and dynamic range all interact to affect perceived image quality. We show how these variables can be combined to reflect the performance trade-offs of electron imaging. Arriving at a single number allows detectors of different hardware and operating principles to be directly compared for their imaging suitability under any desired set of operating conditions.

Joy et al. [1] showed how DQE could be used to compare the performance of electron detectors in terms of their ability to reproduce a calculated imaging signal without adding extra noise. Bok and Schauer [2] expressed DQE as a function of frequency rather than a static parameter. Their method made it clear that a detector's image quality is affected by the frequency response of the detector, because smearing in the image depletes the useful contrast. This also meant that in the SEM, detector performance depends on the scan speed relative to the size of features one wishes to see. Different detectors might be preferable at different scan speeds. Bok and Schauer's method synthesizes both aspects of a detector into a single number.

We build on Bok and Schauer's work in several ways. First, we present a paradox where a detector that has worse DQE at all frequencies can still produce an image with better signal-to-noise ratio (SNR) in specific cases, and discuss why this can make evaluating detector performance difficult. An example is shown in Figure 1. Second, we show how Bok and Schauer's method can be used to produce a single figure of merit that represents the overall quality of a detector. Third, we describe a test sample which allows the spectral DQE figure of merit to be extended to take the dynamic range of the detector into account as well.

Figure 2 illustrates an idealized test sample which has a dark region and a light region, each of which contains local contrast. The large contrast difference between the two regions requires high dynamic range to retain the local contrast. We describe a procedure that uses inexpensive, readily-available materials to approximate such a sample for use in evaluating detector performance for real-world imaging applications, and apply this procedure to several detectors, including recently-developed scintillation detectors that use silicon photomultipliers (SiPMs). Lastly, we suggest ways that these methods could be extended to various operating parameters (e.g. beam current), and what this could mean for choosing the best detector to see a given level of detail under a given set of conditions.

References:

[1] D. C. Joy, C. S. Joy and R. D. Bunn. Scanning 18 (1996) 533-538.

[2] J. Bok and P. Schauer. Scanning 36 (2014) 384-393. 


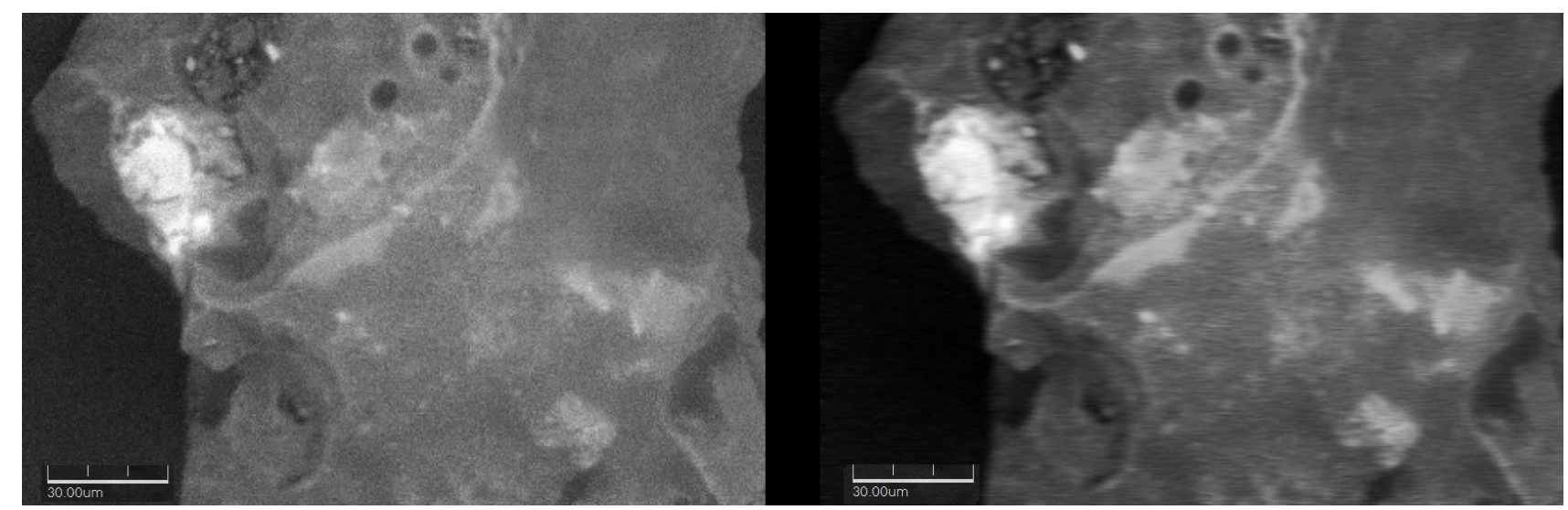

Figure 1: The left image is shown as acquired; the right image has been digitally filtered in the $\mathrm{X}$ axis to simulate smearing from a scan speed too fast for the detector. The image on the left has higher spectral DQE at all frequencies, but the image on the right has higher SNR.
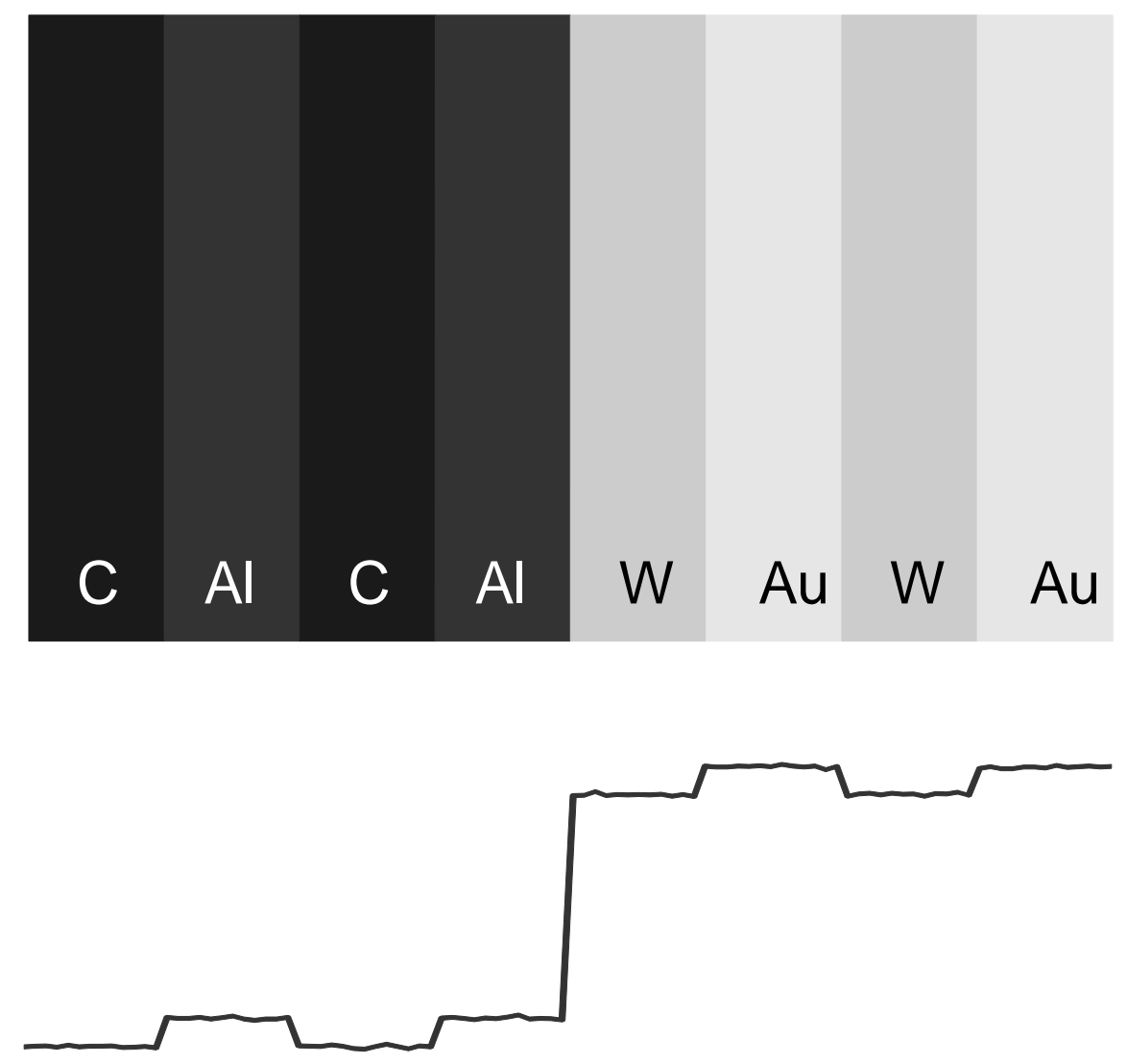

Figure 2: A test sample which challenges dynamic range as well as DQE and bandwidth. 